\title{
A Magnetically Controlled Wireless Optical Oxygen Sensor for Intraocular Measurements
}

\author{
Olgaç Ergeneman, Student Member, IEEE, Görkem Dogangil, Student Member, IEEE, \\ Michael P. Kummer, Student Member, IEEE, Jake J. Abbott, Member, IEEE, Mohammad K. Nazeeruddin, and \\ Bradley J. Nelson, Senior Member, IEEE
}

\begin{abstract}
The influence of oxygen on various ophthalmological complications is not completely understood and intraocular oxygen measurements are essential for better diagnosis and treatment. A magnetically controlled wireless sensor device is proposed for minimally invasive intraocular oxygen concentration measurements. This device will make it possible to make measurements at locations that are currently too invasive for human intervention by integrating a luminescence optical sensor and a magnetic steering system. The sensor works based on quenching of luminescence in the presence of oxygen. A novel iridium phosphorescent complex is designed and synthesized for this system. A frequency-domain lifetime measurement approach is employed because of the intrinsic nature of the lifetime of luminescence. Experimental results of the oxygen sensor together with magnetic and hydrodynamic characterization of the sensor platform are presented to demonstrate the concept. In order to use this sensor for in vivo intraocular applications, the size of the sensor must be reduced, which will require an improved signal-to-noise ratio.
\end{abstract}

Index Terms-In vivo, intraocular, iridium complex, luminescence quenching, magnetic steering, oxygen sensor, sensing.

\section{INTRODUCTION}

$\mathbf{T}$ THE RETINA OF the living eye needs a sufficient supply of oxygen and other nutrients to perform its primary visual function [1]. Inadequate oxygen supply (i.e., retinal hypoxia) is correlated with major eye diseases including diabetic retinopathy, glaucoma, retinopathy of prematurity, age-related macular degeneration, and retinal vein occlusions [2]-[4]. The influence of oxygen on these diseases is not well understood and in vivo oxygen measurements are essential for better diagnosis and treatment.

The ability to make long-term noninvasive oxygen tension measurements in the human eye is a very attractive and essential subject [5]. Measuring the oxygen tensions both in aqueous humor and vitreous humor, and particularly in the preretinal

Manuscript received February 2, 2007; revised April 18, 2007. This work was supported by the NCCR Co-Me of the Swiss National Science Foundation. The Associate Editor coordinating the review of this paper and approving it for publication was Dr. Robert Black.

O. Ergeneman, G. Dogangil, M. P. Kummer, J. J. Abbott, and B. J. Nelson are with the Institute of Robotics and Intelligent Systems, ETH Zurich, 8092 Zurich, Switzerland (e-mail: oergeneman@ethz.ch; gdogangil@student.ethz.ch; kummerm@ethz.ch; jabbott@ethz.ch; bnelson@ethz.ch).

M. K. Nazeeruddin is with the Laboratory for Photonics and Interfaces, Ecole Polytechnique Fédérale de Lausanne, 1015 Lausanne, Switzerland (e-mail: mdkhaja.nazeeruddin@epfl.ch).

Color versions of one or more of the figures in this paper are available online at http://ieeexplore.ieee.org.

Digital Object Identifier 10.1109/JSEN.2007.912552 area, is of great interest in ophthalmological research. Being able to gather this information through a noninvasive method will provide major advantages.

The field of medical robotics bears great potential for improving the capabilities of clinicians when performing critical surgical procedures [6], [7], or when there is a need for precise diagnosis in the living body. During the past decade, the popularity of minimally invasive medical diagnosis and treatment has risen remarkably [8], [9]. In recent years, a great deal of progress has been made in the development of advanced biomedical microdevices, where MEMS technology has enabled the integration of sensors, actuators, and electronic circuits at small scales [10], [11].

Biomicrorobotics is the emerging field combining the principles of robotics with the broad technological innovations of MEMS for use in biomedical applications, such as in vivo minimally or noninvasive diagnosis and treatment. There is strong interest in biomicrorobots that are capable of performing localized sensing or drug delivery in parts of the body that are currently inaccessible or too invasive to access. Energy generation and storage have not yet been successfully miniaturized to the microscale, and transmitting energy using electrical wires is extremely limiting. However, energy transfer to microscale devices can be done wirelessly via externally applied magnetic fields, and there is a significant body of prior work on this topic.

This paper presents the concept and prototype of a minimally invasive wireless optical sensor device (Fig. 1) to measure dissolved oxygen concentration inside the living eye. The device consists of a luminescence sensor film that is integrated with a magnetically controlled platform. The device can be inserted through a small incision in the sclera, and then closed-loop position control within the vitreous humor can be accomplished via applied magnetic fields and visual tracking through the pupil. This system can be used to obtain oxygen concentration maps inside the eye, specifically in the preretinal area, and is also applicable to a number of other analytes of interest (e.g., $\mathrm{pH}$ and glucose).

Reviews of prior work in intraocular oxygen measurement and wireless magnetic manipulation follow directly. Section II describes the optical oxygen sensing technology used in this paper. Section III presents the magnetic control in a fluidic environment. Experimental results of the oxygen sensor are presented in Section IV, including the fabrication of the sensor system and the details of the experimental setup. Section V presents the hydrodynamic and magnetic force measurements of the sensor platform, which are necessary for the control of the sensor. Finally, conclusions are provided in Section VI. 


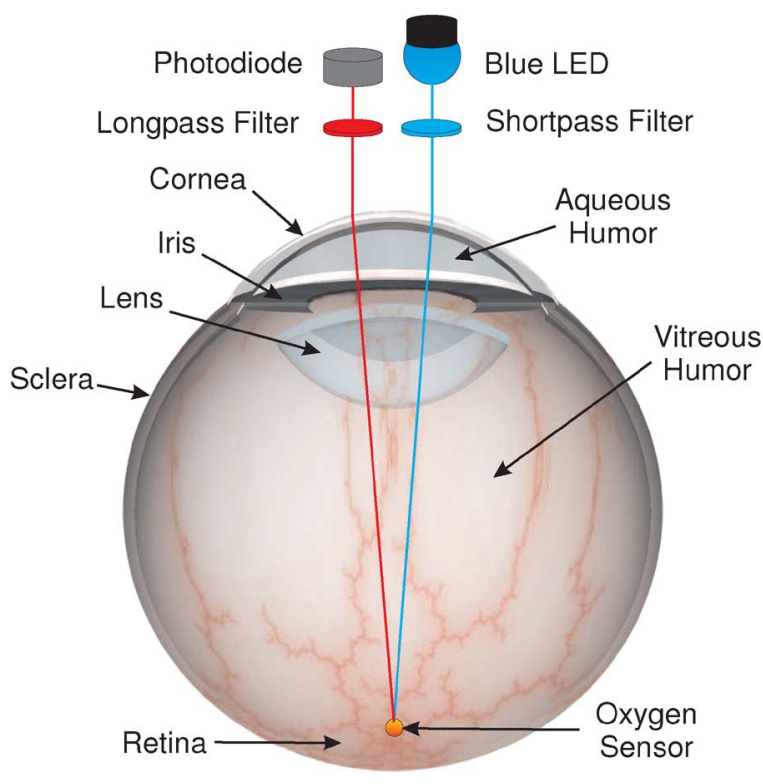

Fig. 1. Artist's conception of wireless oxygen sensor in eye.

\section{A. Prior Work in Measuring Intraocular Oxygen Concentration}

Hypoxia is a pathological condition in which the affected tissues lack adequate oxygen supply. Retinal hypoxia is presumed to initiate angiogenesis, which is a major cause of blindness in developed countries [12]. Attempts to test this hypothesis suffer from the current methods of highly invasive oxygen electrodes. Hypoxia is typically present at the end stages of retinal diseases. However, during the early stages, the relation between blood flow sufficiency, vessel patency, and tissue hypoxia are still unknown [13]. Moreover, the oxygen supply to the cornea and lens of the eye is also of much practical interest in ophthalmological research [14].

To address these issues, intraocular oxygen measurement devices have been developed. The first reliable method used has been the polarographic (Clark) electrode oxygen sensors, which work based on the reduction of oxygen on a noble metal electrode. The electrode is usually surrounded by a membrane that is permeable to molecular oxygen and allows it to reach the electrode where it is electrolytically reduced. The oxygen concentration is proportional to the current resulting from the reduction of oxygen at the surface of this electrode. Despite being accurate, their relative size, slow response time, and motion dependency make them unsuitable for intraocular measurements. This type of electrode also consumes oxygen, which might be problematic for low-oxygen-concentration environments [15]. Moreover, this system is observed to be quite invasive [16]. The need for electrical wiring to the sensor prevents it from being used as a wireless sensor.

Another type of oxygen sensor developed is the fiber-optic probe oxygen sensor based on fluorescence quenching. In this system, fluorescent dyes are packaged into a probe and the oxygen concentration is measured by means of fluorescence quenching, read by another probe that is also inserted in the eye cavity. Unlike polarographic electrode oxygen sensors, the fiber-optic probe oxygen sensors do not consume oxygen [15]. However, they also suffer from excessive invasiveness.
There are promising new noninvasive technologies that measure retinal oxygen concentration indirectly, including those based on magnetic resonance [12], [17] and spectral imaging [18], [19], but they cannot yet provide a quantitative measure of oxygen concentration within the retinal tissue affected by disease. Although another method utilizing phosphorescence is proposed in [13], it requires injecting phosphorescence dyes directly into the eye cavity.

\section{B. Prior Work in Wireless Magnetic Manipulation}

One approach to the wireless control of a sensor platform is through externally applied magnetic fields. There is a significant body of work dealing with noncontact magnetic manipulation [20]. Research has considered the 3-D positioning of permanent magnets [21], [22]. Magnetic fields have been used to orient small permanent magnets placed at the distal tips of catheters [23], [24]. Researchers have considered the position control of soft-magnetic beads as well, where a spherical shape simplifies the control problem [25]. Precision control of nonspherical soft-magnetic bodies has also been considered [26]. In addition to magnetic manipulation of simple objects (e.g., beads, cylinders), it is possible to manipulate more complicated shapes. In [27], a soft-magnetic assembled-MEMS microrobot is controlled by applying decoupled magnetic torque and force. Assembled-MEMS microrobots have the potential to provide increased functionality over simpler geometries. All of the above works generate magnetic fields with custom-built devices, but it is also possible to use the existing field inside an MRI for magnetic control [28].

Surgeons have been using magnets to remove metallic debris from eyes for over 100 years [29]. However, there has been no prior work of controlled magnetic manipulation of an object that has been intentionally inserted in the eye. Intraocular procedures are unique among in vivo procedures, as they provide a direct line of sight through the pupil for visual feedback, making closed-loop control possible.

\section{OPtiCAl Dissolved OXYGEN SENSORS}

Photoluminescence is the emission of optical radiation (i.e., photons) from a material in response to absorption of some form of radiant energy [30]. The intensity and the lifetime of emission can be decreased by a variety of processes. The decrease in intensity and lifetime is called quenching in general. Optical oxygen sensors work based on quenching of luminescence in the presence of molecular oxygen. The quenching of oxygen can be described by Stern-Volmer equations

$$
\begin{aligned}
& \frac{I_{0}}{I}=1+K\left[\mathrm{O}_{2}\right] \\
& \frac{\tau_{0}}{\tau}=1+K\left[\mathrm{O}_{2}\right]
\end{aligned}
$$

where $I_{0}$ and $I$ are the luminescence intensities in the absence and in the presence of oxygen, respectively, $\tau_{0}$ and $\tau$ are the luminescence lifetimes in the absence and presence of oxygen, respectively, $K$ is the Stern-Volmer quenching constant, and $\left[\mathrm{O}_{2}\right]$ is the dissolved oxygen concentration. Luminescence dyes with high quantum yield, large dynamic range, and large Stokes shift are used for luminescence sensors. Ruthenium and iridium 
complexes are examples of such dyes. These dyes are bound to transparent and oxygen-permeable supporting matrices such as polymers, silica gels, or sol-gels. Oxygen permeability and the luminophore solubility of the supporting matrix are two important factors for choosing the appropriate supporting matrix.

\section{A. Sensing Approaches}

Luminescence sensing can be done in four possible ways [30]. The first is the standard intensity-based method in which the signal intensity from the sensing probe changes in response to the analyte of interest (i.e., because of quenching processes). In this type of sensor, the analyte concentration can be related to the signal intensity. This method is the easiest to implement and it has been used for detection of many analytes (e.g., oxygen, iodide, chloride). However, this technique depends on quantities such as the dye concentration, optical surface quality, fluctuations of the excitation source, photo-bleaching, incidence angle, etc. These quantities change from sample to sample, limiting the accuracy of this technique.

The problems of intensity-based measurements can be avoided by using wavelength-ratiometric probes, whose absorption or emission spectrum changes in the presence of an analyte. The concentration of the analyte is found by taking the ratio of intensities at different excitation or emission wavelengths. A disadvantage of this method that limits its usage is the complex probe design.

Lifetime measurements are more promising, since lifetime (unlike intensity) is an intrinsic property, meaning that it does not depend on the extrinsic parameters described above. Emission is a random event so each excited luminophore has the same probability of emitting in a given period of time. This results in an impulse response with an exponential decay in the emission intensity

$$
I(t)=I(0) e^{-t / \tau}
$$

The lifetime of emission decreases in the presence of oxygen as a result of the quenching process. Hence, oxygen concentration can be related to the lifetime of emission. There are two methods that are used for measuring luminescence lifetimes: time-domain measurements and frequency-domain measurements [31]. In time-domain measurements, the sample is excited with light pulses, and the intensity signal that changes as a function of time is measured and analyzed. The time where the intensity decays to $e^{-1}(\sim 36.8 \%)$ of the initial value is the lifetime. In frequency-domain measurements the sample is excited with a periodic signal that consequently causes a modulated luminescence emission at the identical frequency. Because of the lifetime of emission, the emission signal has a phase shift (i.e., time delay) with respect to the excitation signal. The input excitation signal is used as a reference to establish a zero-phase position and the lifetime is obtained by measuring the phase shift between the excitation signal and the emission signal. The relationship between the lifetime $\tau$ and the corresponding phase shift $\phi$ (in radians) for a single exponential decay is given by

$$
\tau=\frac{\tan \phi}{2 \pi f}
$$

where $f$ is the modulation frequency in Hertz.
The intensity of light collected by the photodetector is extremely sensitive to extrinsic conditions, which are hard to control in such a wireless sensor application. While the sensor is steered in the ocular cavity, the optical path distance from the light source to the sensor and back to the photo detector changes. The total amount of light collected by the sensor changes depending on the orientation and location of the sensor as well. Furthermore, there are other parameters that affect the photodetector output as described previously. However, these changes in measurement conditions do not affect the lifetime of emission. Due to its intrinsic nature, the frequency-domain lifetime measurement approach is used in this paper.

\section{MAgnetic CONTROL IN Fluidic ENVIRONMENT}

If the sensor platform is a sphere made of a permanent-magnetic material, where the magnetization of the material $\mathbf{M}$ is essentially independent of the applied magnetic field $\mathbf{H}$, the magnetic force on the sphere is calculated by the force on a magnetic dipole moment [32]

$$
\mathbf{F}_{\text {mag }}=\mu_{0} v(\mathbf{M} \cdot \nabla) \mathbf{H}
$$

where $v$ is the volume of the sphere and $\mu_{0}$ is the permeability of vacuum. Since there is no electric current flowing through the region occupied by the sphere, Maxwell's equations provide the constraint $\nabla \times \mathbf{H}=\mathbf{0}$. This allows us to express (5), after some manipulation, in a more intuitive form

$$
\mathbf{F}_{\text {mag }}=\mu_{0} v\left[\begin{array}{l}
\mathbf{M} \cdot \frac{d}{d x} \mathbf{H} \\
\mathbf{M} \cdot \frac{d}{d y} \mathbf{H} \\
\mathbf{M} \cdot \frac{d}{d z} \mathbf{H}
\end{array}\right]
$$

There is also a magnetic torque on the dipole that tends to align the magnetization $\mathbf{M}$ with the applied field $\mathbf{H}$. With a spherical shape, these rotations are of little interest and are not readily observed visually.

The sphere can also be made of a soft-magnetic material, where the magnetization of the material is largely a function of the applied field, with very little hysteresis. We consider a material with a saturation magnetization value of $m_{s}$ in units of amperes per meter. Because there is no shape anisotropy in a sphere, the magnetization vector $\mathbf{M}$ will always align itself with the applied field $\mathbf{H}$. This leads to a simplification of (5) and (6)

$$
\mathbf{F}_{\text {mag }}=\mu_{0} v|\mathbf{M}| \nabla|\mathbf{H}| .
$$

At relatively low fields, where $|\mathbf{H}|<m_{s} / 3$, the magnetization of a soft-magnetic sphere is linearly related to the applied field

$$
\mathbf{M}=3 \mathbf{H} .
$$

If $|\mathbf{H}|$ is high enough to magnetically saturate the material, then $|\mathbf{M}|=m_{s}$.

Small spheres traveling at slow speeds in viscous fluids operate in a low Reynolds number regime [33], where the viscous drag force is linearly related to the velocity

$$
\mathbf{F}_{\text {drag }}=-3 \pi \eta d \mathbf{V}
$$

where $\eta$ is the fluid viscosity and $d$ is the diameter of the sphere. Equation (9) assumes a sphere in an infinite extent of fluid. 


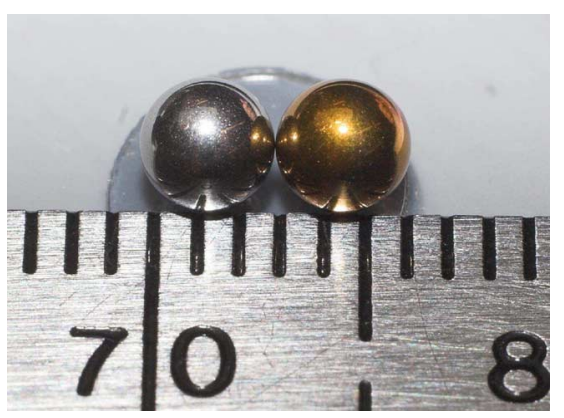

Fig. 2. Uncoated and dip-coated ferromagnetic spheres with diameter of $3.25 \mathrm{~mm}$. Oxygen-sensitive film-coated sphere is on right (orange) and uncoated sphere is on left (metallic). Thin coating has negligible impact on overall sensor geometry.

In real applications, the drag force will always be somewhat higher than predicted by (9). An intraocular sensor must navigate through the vitreous humor, which has a nominal viscosity of $\eta=0.0016 \mathrm{~Pa} \cdot \mathrm{s}$, but varies from person to person [34]. In certain cases, the sensor might encounter fluid pockets in the eye with a viscosity similar to water, $\eta=0.001 \mathrm{~Pa} \cdot \mathrm{s}$.

\section{EXPERIMENTAL RESULTS ON OXYGEN SENSOR}

\section{A. Prototype Sensor}

Ferromagnetic metal spheres of varying size are used for the sensor (Fig. 2). There is a tradeoff between the invasiveness and the performance of the sensor. Large spheres provide highintensity signals and high signal-to-noise ratio. However, the larger the spheres are, the more invasive the procedure becomes. A novel iridium phosphorescent complex $\left[\operatorname{Ir}(2 \text { - phenyl - pyridine })_{2}(4,4-\operatorname{bis}(2-(4-\mathrm{N}, \mathrm{N}-\right.$ methylhexylaminophenyl)ethyl) - $2-2$-bypyridine)Cl], hereafter labeled as N948 for convenience, is designed and synthesized to be used as an oxygen probe. The main advantages of N948 iridium complex, when compared to other metal complexes, are its higher luminescence quantum yield, higher photostability, longer lifetime, stronger absorption band in the visible region, and larger Stokes shift. Polystyrene is chosen as the supporting matrix because of its high oxygen permeability and bio-compatible nature. N948 dyes have a peak excitation wavelength of $494 \mathrm{~nm}$, peak emission wavelength of $665 \mathrm{~nm}$, and luminescence quantum yield of 0.6 in polystyrene.

The N948 complex was synthesized in a low boiling solvent dichloromethane by reacting the dichloro-bridged iridium (III) dimer with $4,4^{\prime}-\operatorname{bis}(2-(4-\mathrm{N}, \mathrm{N}-$ methylhexylaminophenyl)ethenyl) $-2,2^{\prime}-$ bipyridine. In a typical synthesis, the dimeric iridium (III) complex $\left.[\operatorname{Ir}(2-\text { phenyl }) \text { pyridine })_{2}(\mathrm{Cl})\right]_{2}(300 \mathrm{mg} ; 0.28 \mathrm{mM})$ was dissolved in $100 \mathrm{ml}$ of dichloromethane solvent under nitrogen. To this solution $4,4^{\prime}-\operatorname{bis}(2-(4-\mathrm{N}, \mathrm{N}-$ methylhexylaminophenyl)ethenyl) $-2,2^{\prime}$ - bipyridine ( $425 \mathrm{mg}$, $0.724 \mathrm{mM}$ ) was added. The reaction mixture was refluxed under nitrogen for three hours. The solvent dichloromethane was evaporated and the resulting solid was dissolved in $5 \mathrm{~mL}$ of methanol and precipitated by ether. (Yield: $394 \mathrm{mg} ; 63 \%$. Anal. Calcd for $\mathrm{C}_{62} \mathrm{H}_{66} \mathrm{ClIrN}_{6}$ : C, 66.32; H, 5.92; N, 7.48; Found: C, 65.69; $\mathrm{H}, 6.01 ; \mathrm{N}, 7.84$.) The N948 complex was recrystallized from a methanol-ether mixture and characterized by analytical and

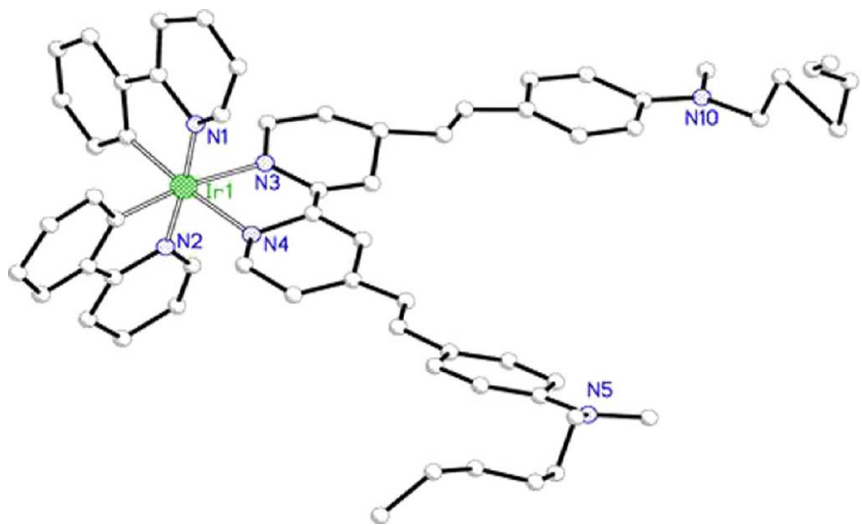

Fig. 3. Molecular drawing of N948 iridium complex.

spectroscopic techniques. The crystal structure of N948 complex clearly shows that the pyridine ligands of 2-phenylpyridine are mutually found in trans position and the phenyl carbons are cis-configuration which are trans to the $4,4^{\prime}-\operatorname{bis}(2-$ $(4-\mathrm{N}, \mathrm{N}-$ methylhexylaminophenyl)ethenyl $)-2,2^{\prime}-$ bipyridine $4,4^{\prime}$ - dimethylamino-2, $2^{\prime}$ - bipyridine ligand. The N948 molecule is shown in Fig. 3. For film preparation, $3 \mathrm{mg}$ of $\mathrm{N} 948$ and $197 \mathrm{mg}$ of polystyrene are dissolved in $2 \mathrm{ml}$ of chloroform by stirring. The spheres are dip coated with this solution and stored for 2 hours, allowing evaporation of chloroform.

\section{B. Sensor Setup}

A blue light-emitting diode (LED) is used as the excitation source for the oxygen sensor system. A function generator drives the LED circuit with a sinusoidal signal at $1000 \mathrm{~Hz}$. This frequency is chosen to maximize the output signal. A short-pass optical filter $\left(\lambda_{\text {cutoff }}=500 \mathrm{~nm}\right.$, FES0500, Thorlabs $\mathrm{GmbH})$ serves to limit the spectrum of the excitation to short wavelengths. A long-pass optical filter $\left(\lambda_{\text {cutoff }}=550 \mathrm{~nm}\right.$, FEL0550, Thorlabs $\mathrm{GmbH}$ ) and a photodiode (PD) serve to detect the luminescence, which occurs at longer wavelengths. The transmission characteristics of the filters and the emission spectrum of N948 dyes are obtained using a spectrometer (AvaSpec-2048, Avantes, Inc.). They are shown in Fig. 4. The setup to obtain the spectrometer data is shown in Fig. 5. A commercial large area amplified Si photodetector in an aluminum housing (PDA100A-EC, Thorlabs $\mathrm{GmbH}$ ) is used to obtain high photo sensitivity and low noise. Using a data acquisition card (NI PCI-6259, National Instruments Corporation), the amplified signal is read by the computer, as is the input modulating signal, which is used as a reference. The signals are digitally band-pass filtered to eliminate the noise at other frequencies, as well as the dc components of the signals. The data acquisition is done at a rate of 500000 samples per second. This provides a resolution of $2 \mu \mathrm{s}$. To improve the time resolution of the system, a sine fitting algorithm is applied using MATLAB. The fitting algorithm was tested using an electronic delay circuit and a resolution in the order of $10 \mathrm{~ns}$ was obtained experimentally.

\section{Calibration Setup}

The experimental setup used to characterize the oxygen sensitivity of the sensor is shown in Figs. 6 and 7. Deionized 


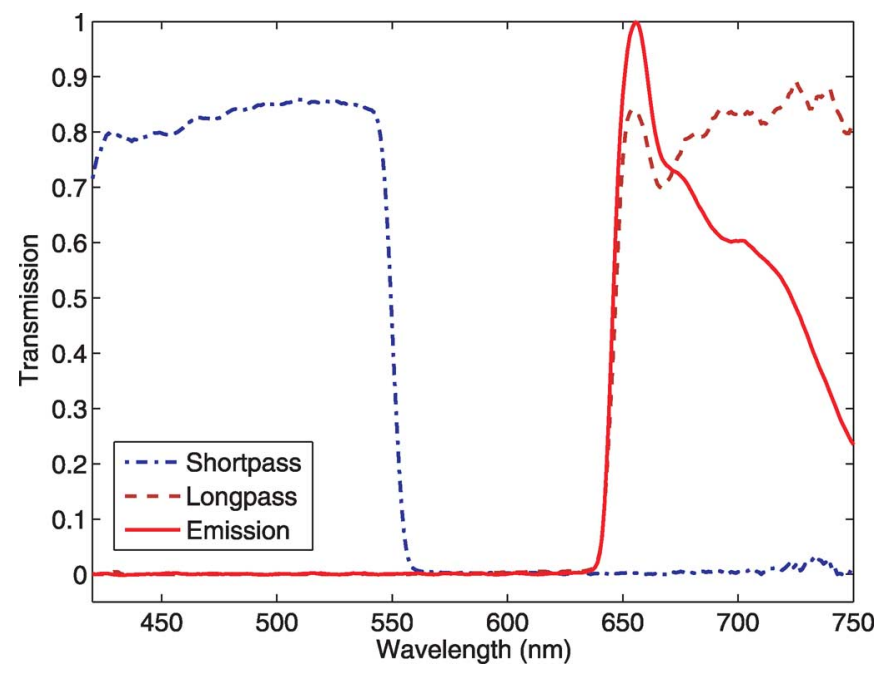

Fig. 4. Experimental data showing transmission characteristics of long-pass and short-pass filters and emission spectrum of N948 polystyrene matrix.

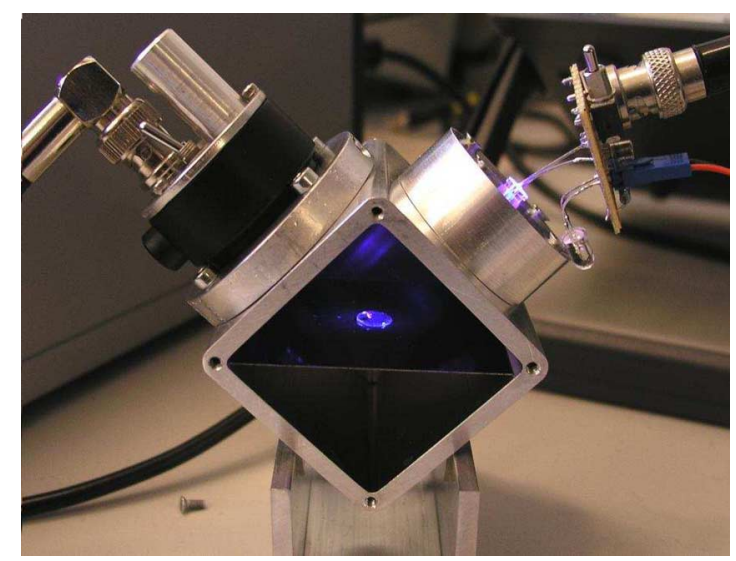

Fig. 5. Setup for emission spectrum measurements. Sphere coated with luminescence film can be seen inside setup.

water is used for the dissolved oxygen measurements. Two PMMA containers, a PMMA pipe connecting them, and a valve provide a sample-and-hold configuration. PMMA was chosen as the material because of its transparency in the UV spectrum, which makes it superior to glass for this application. Furthermore, PMMA has a very low gas permeability $(0.032$ and 0.0039 Barrers for oxygen and nitrogen, respectively [35]), which makes it effectively an impermeable barrier and keeps the gas concentration as anticipated throughout the experiment. The sensor sphere is immersed in the pipe for the measurements and its location is maintained with a permanent magnet. The excitation and readout components are placed at different sides of the pipe. The distances between the components and the sensor are chosen considering the geometry of the eye. The average distance between the cornea and the retina of the human eye is $22.2 \mathrm{~mm}$ [36]. Therefore, the distance between the setup components and the sensor was kept larger than $22.2 \mathrm{~mm}$ throughout the experiments.

One of the containers is filled with water and a range of oxygen concentrations is achieved by bubbling air or nitrogen gas inside this container. Nitrogen replaces oxygen molecules

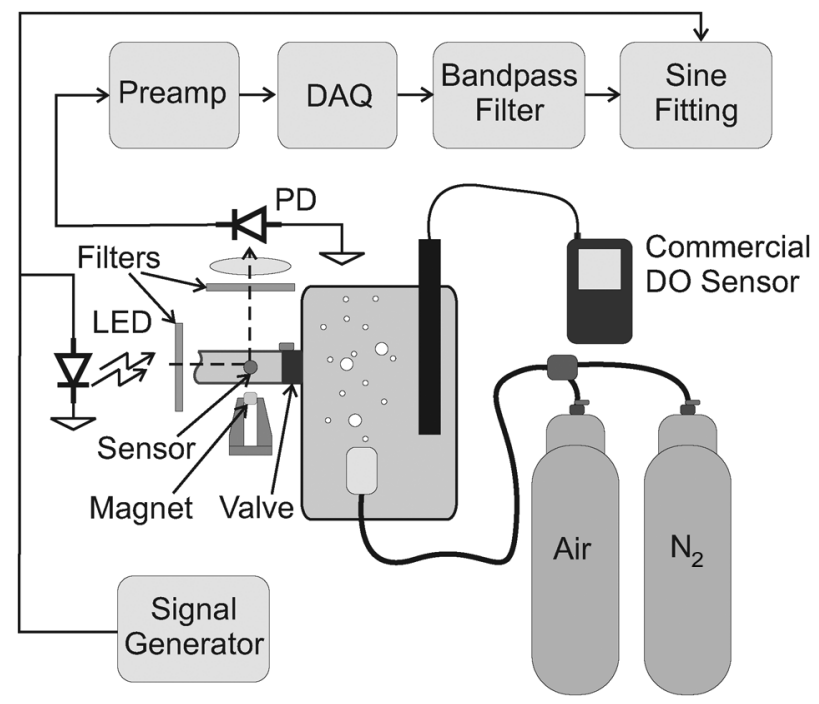

Fig. 6. Schematic of experimental setup for oxygen concentration measurements. Half of pipe and container used as sink are not shown in figure for simplicity.
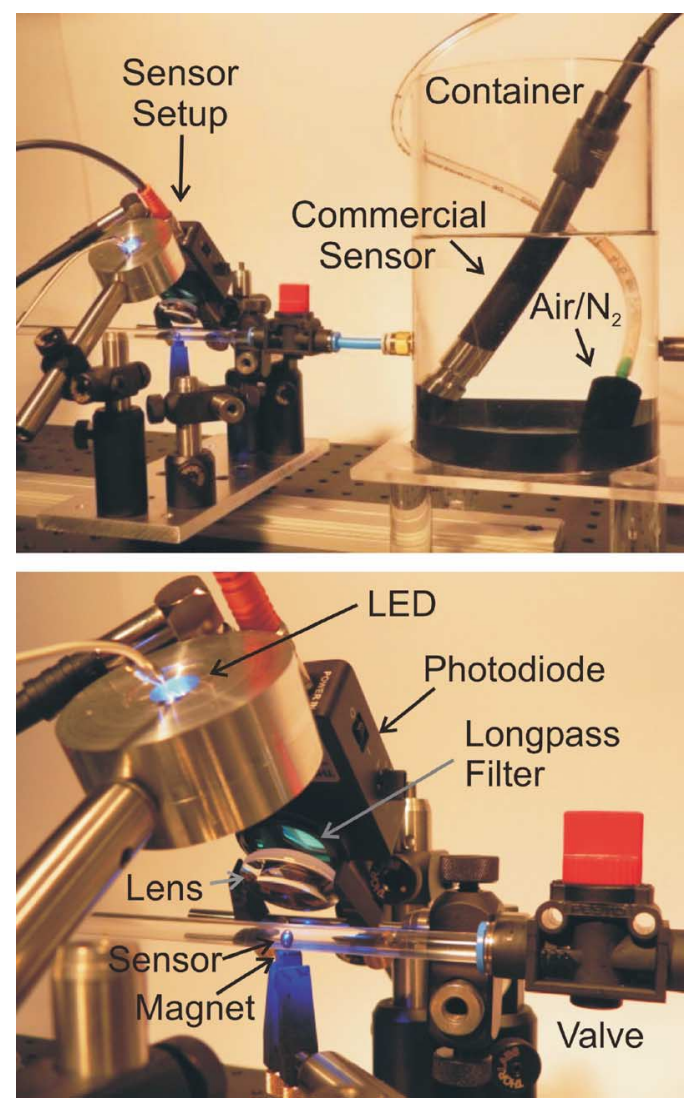

Fig. 7. Photo of experimental setup for oxygen concentration measurements.

in the solution while air provides oxygen. Consequently, by applying nitrogen the oxygen concentration can be reduced, and by applying air it can be increased up to air saturation level at a given pressure and temperature condition. A commercial electrochemical oxygen sensor (Oxi340i, WTW $\mathrm{GmbH}$ ) is used as a reference, which was calibrated at the start of each experiment using its calibration vessel. While bubbling gases into the container, the oxygen concentration is monitored using this sensor. 


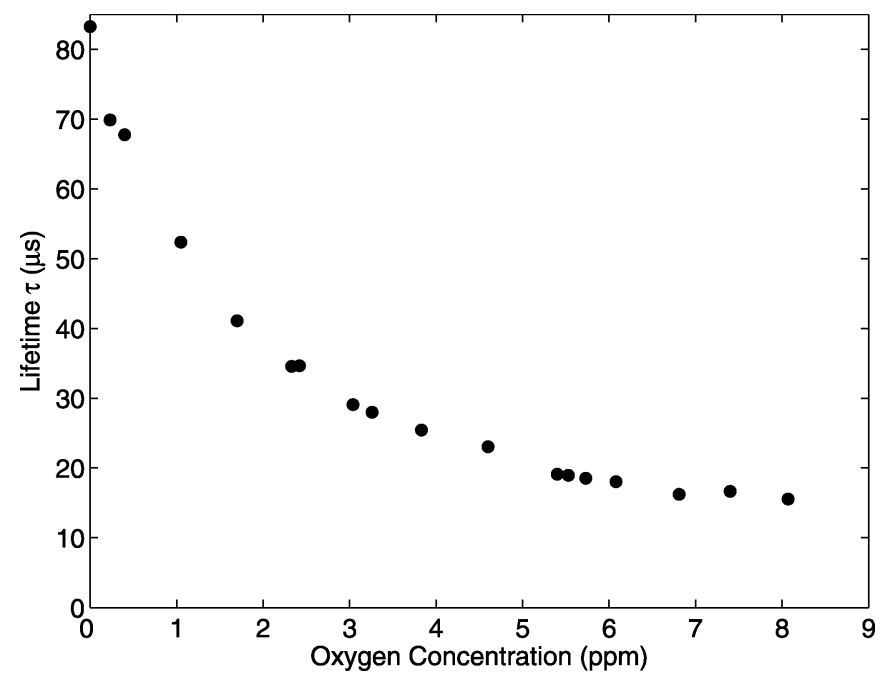

Fig. 8. Lifetime of N948 dyes immobilized in polystyrene film under various oxygen concentrations.

When the desired concentration is reached, the valve of the pipe connecting the two containers is opened to fill the pipe with this solution at known oxygen concentration and then closed again. This makes a simple sample-and-hold configuration for the measurement. The second container is used as a sink for the solution. At the time scales of this experiment, the effect of diffusion on the oxygen concentration inside the pipe is negligible.

\section{Oxygen Concentration Measurements}

The lowest and highest oxygen concentration levels achieved with the method described previously were 0.25 and $8.27 \mathrm{ppm}$, respectively. Three cycles were made going from the lowest concentration level to the highest and back to the lowest again. Seventeen measurements were taken at different oxygen concentration levels without the presence of ambient light. For each measurement the gas flow was stopped, the concentration was measured using the commercial sensor, and the solution was sampled and held with the valve. The lifetime measurement with the sensor was then performed. Fig. 8 shows the lifetime of emission as a function of oxygen concentration. The inherent nonlinear dependence of the quenching process on oxygen concentration, predicted by (2), is obvious in this figure. Fig. 9 shows the Stern-Volmer plot for the same data. As seen in this figure, a linear model proved to be an excellent predictor $\left(R^{2}=\right.$ 0.989 ) for oxygen concentrations compared to the commercial sensor. The model predicts the Stern-Volmer constant as $K=$ $0.567 \mathrm{ppm}^{-1}$.

Fig. 10 shows the lifetime of emission of another sensor sphere as a function of oxygen concentration and the standard deviation of the lifetime for ten consecutive measurements. The standard deviation is smaller for low oxygen concentrations. This is because of the change in intensity. The intensity decreases because of quenching at higher oxygen concentrations. The noise of the system does not change as a function of oxygen concentration. Therefore, the signal-to-noise ratio decreases as oxygen concentration increases, and consequently, the standard deviation increases as well. By comparing Figs. 8 and 10, we

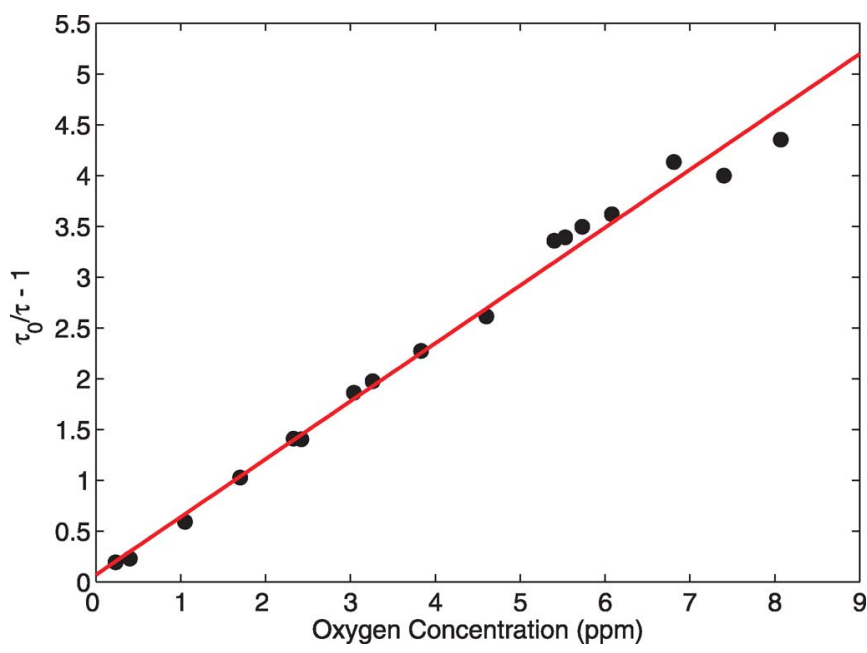

Fig. 9. Stern-Volmer plot of N948 dyes immobilized in polystyrene film under various oxygen concentrations.

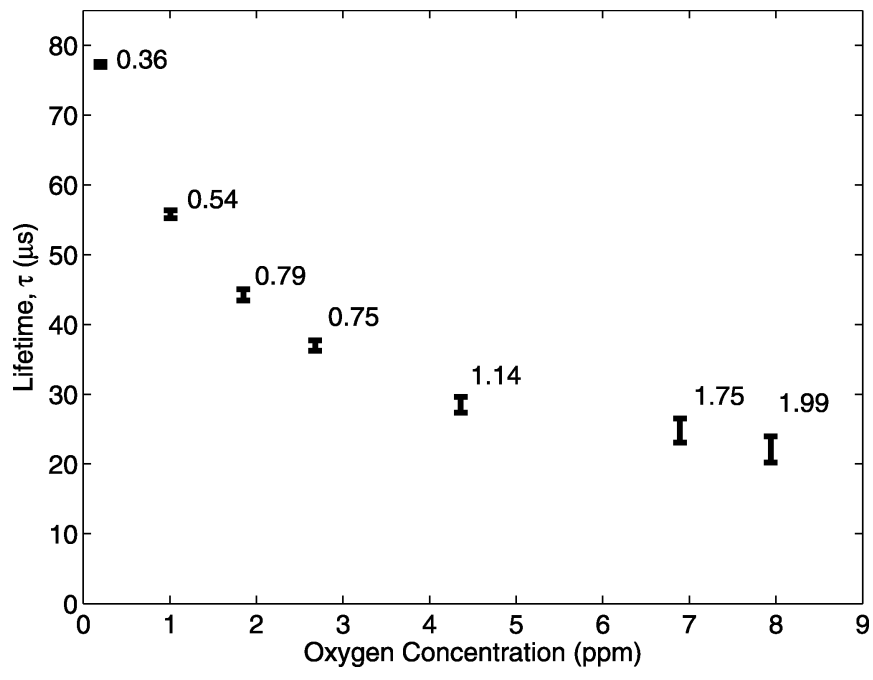

Fig. 10. Lifetime of sensor under various oxygen concentrations. Error bars show standard deviation for ten consecutive measurements.

can see the variation of sensor output between different sensors that were fabricated with the same techniques.

\section{EXPERIMENTAL RESUlTS ON MAGNETIC CONTROL}

For precise sensor control, the behavior of the magnetic sensor platform-being driven by external magnetic fields through various fluid environments, including those which are non-Newtonian - must be characterized. In order to verify the models given in Section III, the magnetic and hydrodynamic properties of metal spheres, which are used as the sensor platform, are obtained experimentally. A custom-built experimental measurement system that is described in detail in [37] is used to investigate magnetic force and hydrodynamic properties of the sensor platform throughout the remainder of this section.

\section{A. Magnetic Force Measurements}

First, the magnetic force on metal spheres is measured as a function of the applied magnetic field. A strong permanent 


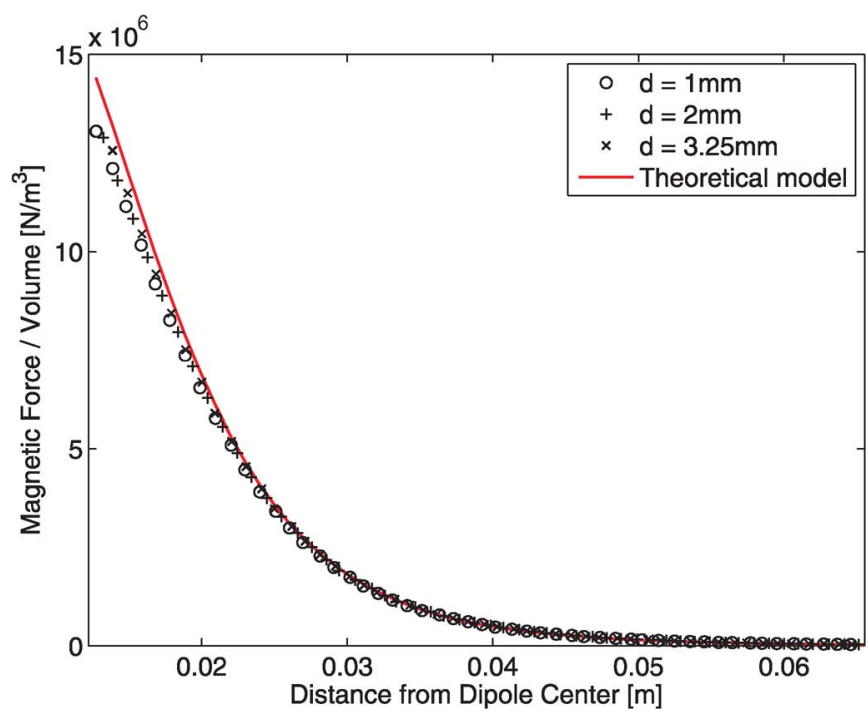

Fig. 11. Magnetic force on three spheres with diameters of 1,2, and $3.25 \mathrm{~mm}$ as a function of displacement between dipole and center of sphere.

magnet with a size of $40 \times 40 \times 20 \mathrm{~mm}$ and with the north and south poles located on the two largest faces is used to generate the external magnetic field. The magnetic field of the permanent magnet along its dipole axis is measured before the experiment, so the magnetic field intensity, direction, and gradient are well known and characterized. To model the field along the dipole axis of this magnet, a current loop model is used, where the dipole is created by a current loop of radius $r$ with current $i$, resulting in a magnetic moment $|\boldsymbol{\Gamma}|=\pi r^{2} i$ with the direction of $\Gamma$ defined by the right-hand rule. The magnitude of the field along the axis of the loop can be calculated with

$$
|\mathbf{H}|=\frac{|\mathbf{\Gamma}|}{2 \pi\left(r^{2}+|\mathbf{P}|^{2}\right)^{3 / 2}}
$$

in units of amperes per meter, where $\mathbf{P}$ is the vector from the dipole to the position of interest. The model is accurately fit to the measured data set $\left(\mathrm{R}^{2}=0.999\right)$ with $\Gamma=29.36 \mathrm{~A} / \mathrm{m}^{2}, r=$ $22.83 \mathrm{~mm}$ and the center of the current loop located $7.251 \mathrm{~mm}$ below the magnet surface. Once equipped with a differentiable model of the magnetic field along the dipole axis, the gradient along this axis can also be calculated.

The experimental setup is used to measure the magnetic force on three different steel spheres with diameters of 1,2 , and $3.25 \mathrm{~mm}$. The spheres are mounted to a long, thin plexiglass rod that is hung from an Ohaus Analytica Plus high precision scale. The magnet is placed under the sphere with its dipole axis aligned with the thin rod. The force data is taken while the magnet is moved along its axis. Fig. 11 shows the measured magnetic force per volume on the three spheres as a function of distance between the dipole and the center of the spheres. The forces predicted using (7) and (10) are shown as well. The model is an accurate predictor of magnetic force. As the field becomes very large, we observe saturation effects in the experimental data that are not predicted by the simple linear (i.e., constant susceptibility) model, making the model slightly overpredict the magnetic force.

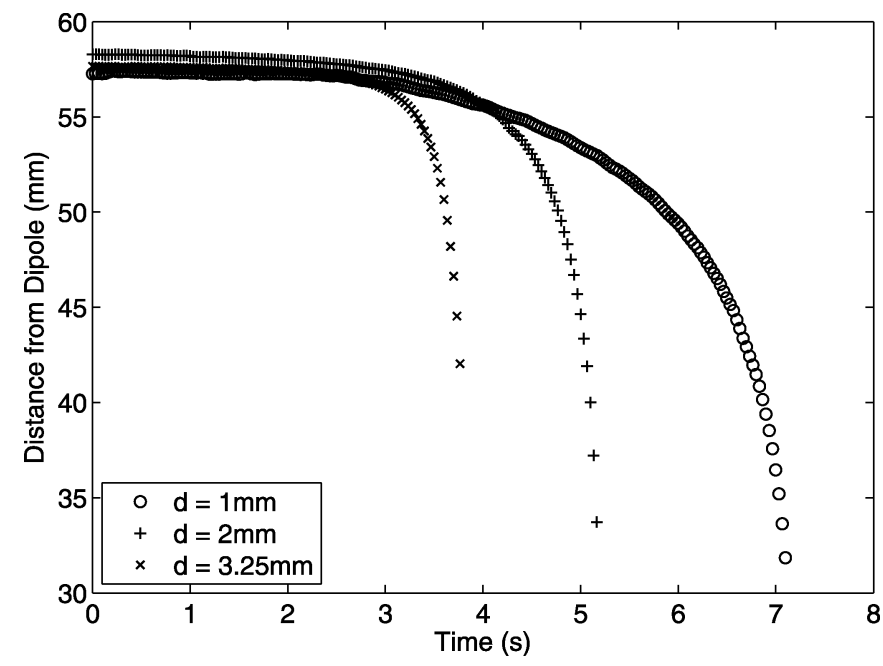

Fig. 12. Distance plotted as a function of time for three spheres with diameters of 1,2 , and $3.25 \mathrm{~mm}$ when spheres are pulled towards magnet through AK1000 Silicon oil.

\section{B. Measuring Hydrodynamic Properties}

In order to understand the relationship between applied magnetic force and velocity, the hydrodynamic properties of the sensor platform must be obtained. We are interested in small slow-moving spheres in a fluid environment. They operate at a low Reynolds number, where inertial effects are negligible and viscous effects dominate. As a worst case estimate, a $3.25-\mathrm{mm}$ sphere moving inside water with a velocity of $2 \mathrm{~mm} / \mathrm{s}$ will have $\operatorname{Re}=6.5$. At low Reynolds numbers, the sphere can be assumed to reach its terminal velocity nearly instantaneously, where the viscous drag force balances the net applied-magnetic and gravity/buoyancy forces.

In order to obtain the hydrodynamic properties, the sensor platform is placed at the bottom of a vial filled with AK1000 silicon oil (density: $970 \mathrm{~kg} / \mathrm{m}^{3}$, viscosity: $0.98 \mathrm{~Pa} \cdot \mathrm{s}$ at $25^{\circ} \mathrm{C}$ ). The vial is positioned underneath the magnet so that the center of the sphere is aligned with the dipole axis of the magnet, yet the attractive magnetic force does not overcome gravity. The position of the magnet is controlled vertically using a linear stage and the position of the sphere is tracked with two Basler A602f digital cameras oriented orthogonally to one another. The gap between the magnet surface and the bottom of the vial is reduced to a constant distance in order to pull the sphere towards the magnet. In Fig. 12, vertical displacement of the spheres with three different sizes are plotted as a function of time. The closer a sphere gets to the surface, the stronger the force acting on the sphere.

In Fig. 13, the net force (applied-magnetic and gravity/buoyancy forces) acting on the sphere measured in Section V-A, normalized with respect to the sphere diameter $d$, is plotted against velocity, which is obtained by numerical differentiation of the displacement data with respect to time. A three-point-movingaverage smoothing filter was applied to the original displacement data before differentiation. The theoretical curve based on (9) is plotted in Fig. 13 as well. The differences between the experimental data sets and the theoretical line is due to the limited size of the vial. The equations assume an infinite extent of 


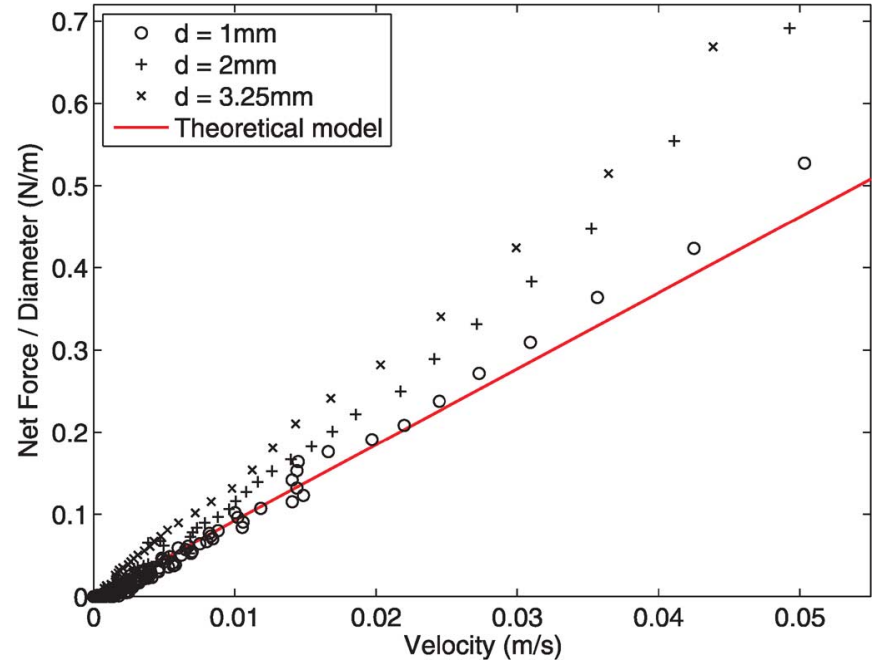

Fig. 13. Net force (applied-magnetic and gravity/buoyancy forces) normalized with respect to diameter plotted as a function of velocity for three spheres with diameters of 1,2 , and $3.25 \mathrm{~mm}$.

fluid but the experiments are done in a relatively small vial with a diameter of $30 \mathrm{~mm}$. The discrepancy between the theoretical model and the observed behavior becomes more significant as the ratio of the diameter of the sphere to the diameter of vial increases. These effects will be mitigated by a reduction in sphere size. However, when the sphere nears a surface, the actual velocity will always be smaller than that predicted by (9), for a given magnetic force, due to increases in shear force resulting from wall effects.

\section{CONCLUSION}

A magnetically controlled wireless optical oxygen sensor is presented. The sensor provides a minimally invasive alternative method to intraocular oxygen concentration measurements and paves the way for development of miniaturized wirelessly controlled sensors. The concept of integrating luminescence sensors with wirelessly controlled magnetic spheres can be extended. Different dye-supporting matrix combinations can be developed to investigate other physical and chemical properties (e.g., temperature, $\mathrm{pH}$ ) or analytes of interest (e.g., glucose, $\mathrm{CO}_{2}$ ). Custom shapes and materials can also be used to optimize the steering capabilities of the sensor and to allow precise control. Concentration maps can be obtained by steering the sensor and taking measurements at many different points of interest. With its magnetic steering system, this sensor system bears great potential for ophthalmologic research and diagnosis.

In order to use this sensor for in vivo intraocular applications, the size of the sensor must be reduced (i.e., diameter below $1 \mathrm{~mm})$. There is no difficulty in scaling this sensor down to micro dimensions in terms of fabrication. However, the resolution of the sensor is a function of the signal-to-noise ratio. The development of improved luminescence dyes and dye-supporting matrix combinations are active areas of research. Newer dyes promise increased quantum yield, allowing equivalently strong signals from increasingly smaller packages.

\section{ACKNOWLEDGMENT}

The authors would like to thank Dr. J. F. Fernández Sánchez from the Department of Analytical Chemistry, University of Granada, Spain, for his assistance in preparation of the sensor, Dr. med. J. Garweg, the Director of the Swiss Eye Institute, Bern, Switzerland, for his guidance on vitreoretinal applications of biomicrorobots, and K. Vollmers and C. Bergeles from the Institute of Robotics and Intelligent Systems, ETH Zurich, for help in the experimental work and in creating Fig. 1, respectively.

\section{REFERENCES}

[1] D.-Y. Yu and S. J. Cringle, "Oxygen distribution and consumption within the retina in vascularised and avascular retinas and in animal models of retinal disease," Progress Retinal Eye Res., vol. 20, no. 2 , pp. 175-208, 2001.

[2] E. Stefansson, "Oxygen and diabetic eye disease," Graefe's Arch. Clin. Exp. Ophthalmol., vol. 228, pp. 120-123, 1990.

[3] L. Smith, E. Wesolowski, A. McLellan, S. K. Kostyk, R. D’Amato, R. Sullivan, and P. A. D'Amore, "Oxygen induced retinopathy in the mouse,” Invest. Ophthalmol. VisualSci., vol. 35, pp. 101-111, 1994.

[4] N. R. Galloway, W. M. K. Amoaku, P. H. Galloway, and A. C. Browning, Common Eye Diseases and Their Management, 3rd ed. London, U.K.: Springer, 2005.

[5] C. A. Wilson, B. A. Berkowitz, B. W. McCuen, 2nd, and H. C. Charles, "Measurement of preretinal oxygen tension in the vitrectomized human eye using fluorine-19 magnetic resonance spectroscopy," $J$. Appl. Physiol., vol. 110, no. 8, pp. 1098-1100, 1992.

[6] K. Cleary and C. Nguyen, "State of the art in surgical robotics: Clinical applications and technology challenges," Computer-Aided Surgery, vol. 6 , no. 6 , pp. 312-328, 2001.

[7] R. H. Taylor and D. Stoianovici, "Medical robotics in computer-integrated surgery," IEEE Trans. Robotics Automat., vol. 19, no. 5, pp. 765-781, Oct. 2003.

[8] 2007, Given Imaging. [Online]. Available: http://www.givenimaging. $\mathrm{com} /$

[9] 2007, Intuitive Surgical. [Online]. Available: http://www.intuitivesurgical.com/

[10] P. Dario, M. C. Carrozza, A. Benvenuto, and A. Menciassi, "Micro-systems in biomedical applications," J. Micromechanics Microeng., vol. 10, pp. 235-244, 2000.

[11] Y. Haga and M. Esashi, "Biomedical microsystems for minimally invasive diagnosis and treatment," Proc. IEEE, vol. 92, no. 1, pp. 98-114, Jan. 2004.

[12] B. Berkowitz and C. Wilson, "Quantitative mapping of ocular oxygenation using magnetic resonance imaging," Magn. Reson. Med., vol. 33, no. 4, pp. 579-581, 1995.

[13] R. Shonat and A. Kight, "Oxygen tension imaging in the mouse retina," Ann. Biomedical Eng., vol. 31, pp. 1084-1096, 2003.

[14] M. Kwan, J. Niinikoski, and T. Hunt, "In vivo measurements of oxygen tension in the cornea, acqueous humor, and anterior lens of the open eye," Investigative Ophthalmology Visual Sci., vol. 11, pp. 108-114, 1972.

[15] E. Stefansson, J. Peterson, and Y. Wang, "Intraocular oxygen tension measured with a fiber-optic sensor in normal and diabetic dogs," Amer. J. Physiol. Heart Circ. Physiol., vol. 256, pp. 1127-1133, 1989.

[16] E. Stefansson, R. Novack, and D. Harchell, "Vitrectomy prevents retinal hypoxia in branch retinal vein occlusion," Investigative Ophthalmology Visual Sci., vol. 31, no. 2, pp. 284-289, 1990.

[17] T. Duong, S.-C. Ngan, K. Ugurbil, and S.-G. Kim, "Functional magnetic resonance imaging of the retina," Invest. Ophthalmol. Visual Sci., vol. 43, pp. 1176-1181, 2002.

[18] J. Beach, K. J. Schwenzer, S. Srinivas, D. Kim, and J. S. Tiedeman, "Oximetry of retinal vessels by dual-wavelength imaging: Calibration and influence of pigmentation," J. Appl. Physiol., vol. 86, pp. 748-758, 1999.

[19] T. Bonhoeffer and A. Grinvald, "Optical imaging based on intrinsic signals: The methodology," in Brain Mapping: Methods, A. W. Toga and J. C. Mazziotta, Eds. San Diego, CA: Academic, 1996, pp. 55-97.

[20] G. T. Gillies, R. C. Ritter, W. C. Broaddus, M. S. Grady, M. A. Howard, III, and R. G. McNeil, "Magnetic manipulation instrumentation for medical physics research," Rev. Scientific Instruments, vol. 65, no. 3, pp. 533-562, 1994. 
[21] M. S. Grady, M. A. Howard, III, J. A. Molloy, R. C. Ritter, E. G. Quate, and G. T. Gillies, "Nonlinear magnetic stereotaxis: Three-dimensional, in vivo remote magnetic manipulation of a small object in canine brain," Medical Phys., vol. 17, no. 3, pp. 405-415, 1990.

[22] M. B. Khamesee, N. Kato, Y. Nomura, and T. Nakamura, "Design and control of a microrobotic system using magnetic levitation," IEEE/ ASME Trans. Mechatronics, vol. 7, no. 1, pp. 1-14, Jan. 2002.

[23] I. Tunay, "Modeling magnetic catheters in external fields," in Proc. IEEE Int. Conf. Eng. Medicine and Biology Soc., 2004, pp. 2006-2009.

[24] 2007, Stereotaxis [Online]. Available: http://www.stereotaxis.com/

[25] F. Amblard, B. Yurke, A. Pargellis, and S. Leibler, "A magnetic manipulator for studying local rheology and micromechanical properties of biological systems," Rev. Scientific Instruments, vol. 67, no. 3, pp. 818-827, 1996.

[26] J. J. Abbott, O. Ergeneman, M. P. Kummer, A. M. Hirt, and B. J. Nelson, "Modeling magnetic torque and force for controlled manipulation of soft-magnetic bodies," IEEE Trans. Robotics, to be published.

[27] K. B. Yesin, K. Vollmers, and B. J. Nelson, "Modeling and control of untethered biomicrorobots in a fluidic environment using electromagnetic fields," Int. J. Robotics Res., vol. 25, no. 5-6, pp. 527-536, 2006.

[28] J.-B. Mathieu, G. Beaudoin, and S. Martel, "Method of propulsion of a ferromagnetic core in the cardiovascular system through magnetic gradients generated by an MRI system," IEEE Trans. Biomed. Eng., vol. 53, no. 2, pp. 292-299, Feb. 2006.

[29] M. Bach, M. Oschwald, and J. Röver, "On the movement of an iron particle in a magnetic field," Documenta Ophthalmologica, vol. 68, pp. 389-394, 1988.

[30] J. R. Lakowicz, Principles of Fluorescence Spectroscopy, 2nd ed. New York: Kluwer/Plenum, 1999.

[31] C. McDonagh, C. Kolle, A. K. McEvoy, D. L. Dowling, A. A. Cafolla, S. J. Cullen, and B. D. MacCraith, "Phase fluorometric dissolved oxygen sensor," Sensors Actuators B, vol. 74, pp. 124-130, 2001

[32] D. Jiles, Introduction to Magnetism and Magnetic Materials. London, U.K.: Chapman and Hall, 1991.

[33] J. Happel and H. Brenner, Low Reynolds Number Hydrodynamics. The Hague: Martinus Nijhoff, 1983.

[34] T. V. Chirila and Y. Hong, "The vitreous humor," in Handbook Biomaterial Properties, J. Black and G. Hastings, Eds. London, U.K.: Chapman and Hall, 1998, pp. 125-131.

[35] K. E. Min and D. R. Paul, "Effect of tacticity on permeation properties of poly (methyl methacrylate)," J. Polymer Sci. Part B Polymer Phys., vol. 26, pp. 1021-1033, 1988.

[36] C. W. Oyster, The Human Eye: Structure and Function. Sunderland, MA: Sinauer, 1999.

[37] M. P. Kummer, J. J. Abbott, K. Vollmers, and B. J. Nelson, "Measuring the magnetic and hydrodynamic properties of assembled-MEMS microrobots," in Proc. IEEE Int. Conf. Robotics Automation, 2007, pp. $1122-1127$.

Olgaç Ergeneman (S'04) received the M.S. degree in electrical engineering from Koç University, Istanbul, Turkey, in 2005. He is working toward the Ph.D. degree at the Institute of Robotics and Intelligent Systems, ETH Zurich, Zurich, Switzerland, where he is developing biomedical MEMS sensors and actuators.
Görkem Dogangil ( $\mathrm{S}^{\prime} 05$ ) received the B.S. degree in mechanical engineering from Istanbul Technical University, Istanbul, Turkey, in 2005. He is working toward the M.S. degree at the Institute of Robotics and Intelligent Systems, ETH Zurich, Zurich, Switzerland, where his research involves biomicrorobots.

Michael P. Kummer (S'07) received the M.S. degree in mechanical engineering from ETH Zurich, Zurich, Switzerland, in 2005. He is working toward the Ph.D. degree at the Institute of Robotics and Intelligent Systems, ETH Zurich, where he is investigating the control of biomicrorobots.

Jake J. Abbott (M'06) received the Ph.D. degree in mechanical engineering from Johns Hopkins University, Baltimore, MD, in 2005.

$\mathrm{He}$ is a Postdoctoral Research Associate at the Institute of Robotics and Intelligent Systems, ETH Zurich, Zurich, Switzerland, where he is developing biomicrorobots.

Mohammad K. Nazeeruddin received the Ph.D. degree in inorganic chemistry from Osmania University, Hyderabad, India, in 1986.

He joined Deccan College of Engineering and Technology, Osmania University, as a Lecturer. He then moved to Central Salt and Marine Chemicals Research Institute, Bhavnagar, as a Research Associate. He is currently a Senior Scientist in the Laboratory for Photonics and Interfaces, Ecole Polytechnique Fédérale de Lausanne, Lausanne, Switzerland, where his research focuses on dye-sensitized solar cells, hydrogen production, light-emitting diodes, and chemical sensors.

Bradley J. Nelson (SM'06) received the Ph.D. degree in robotics from Carnegie Mellon University, Pittsburgh, PA, in 1995.

He became an Assistant Professor at the University of Illinois, Chicago, in 1995, Associate Professor at the University of Minnesota, in 1998, and Professor of Robotics and Intelligent Systems, ETH Zurich, Zurich, Switzerland, in 2002. He is currently the Head of the Multi-Scale Robotics Laboratory, Institute of Robotics and Intelligent Systems, ETH Zurich, where his research involves microrobotics and nanorobotics. 